\title{
Aperçu Sur La Place De La Demarche D’investigation En Sciences Experimentales Dans L'enseignement Du Secondaire Qualifiant Cas De L'academie De La Ville De Fes
}

\author{
Ihsane Kouchou, PhD \\ Fatiha Kaddari, Prof. \\ Nezha Bennis, Prof. \\ Rida Hajji Hour, PhD \\ Asmae Bouayad, PhD \\ Faculté des Sciences Dhar El Mehraz, Fès/ \\ Laboratoire de Didactique d'Innovation \\ Pédagogique et Curriculaire (LADIPEC), Maroc
}

doi: 10.19044/esj.2017.v13n7p159 URL:http://dx.doi.org/10.19044/esj.2017.v13n7p159

\begin{abstract}
The investigative approach (DI) demonstrates more and more its potential in teaching. Indeed, it enables students to develop skills, attitudes and interests, which are necessary to live in a society increasingly dependent on the applications of science. This study falls within the framework of research studies questioning the application of the investigative approach in the teaching of science in Morocco. In this work, we aim to highlight the representations of secondary school teachers about the concept of investigative approach, see whether this approach is adopted or not in the teaching of experimental sciences disciplines (Science of life and earth, Science of physics and chemistry) and determine the constraints and the obstacles to its implementation. In order to achieve these objectives, a questionnaire was drawn up and was the subject of an exploratory study among 45 teachers of experimental sciences practicing in different secondary schools in Fez city. The results of this survey reveal, firstly, that the teachers questioned seem to have confusions about the investigation process and secondly, they highlight the presence of a set of constraints and difficulties which prevent its application in the classroom.
\end{abstract}

Keywords: Investigative approach, experimental sciences, misconceptions 


\section{Résumé}

La démarche d'investigation (DI) démontre de plus en plus son potentiel en enseignement. En effet, elle permet aux élèves de développer des compétences, des attitudes et des intérêts, nécessaires pour vivre dans une société de plus en plus dépendante des applications des sciences. Cette étude s'inscrit dans le cadre des travaux de recherche s'interrogeant sur l'application de la démarche d'investigation dans l'enseignement des sciences au Maroc. Par ce travail nous visons mettre en exergue les représentations des enseignants du secondaire qualifiant sur le concept de la DI, voir si cette démarche est adoptée ou non dans l'enseignement des disciplines des sciences expérimentales (Sciences de la vie et de la terre, Sciences de la physique et de la chimie) et enfin déterminer les contraintes et les obstacles à sa mise en pratique. Afin de réaliser ces objectifs, une étude exploratoire via un questionnaire, a été menée auprès de 45 enseignants de sciences expérimentales exerçants dans différents Lycée de la ville de Fès. Les résultats de cette enquête, révèlent dans un premier temps que les enseignants questionnés semblent avoir des confusions relatives à la démarche d'investigation et dans un seconde temps ils mettent en évidence la présence d'un ensemble de contraintes et difficultés qui entravent son application en classe.

Mots clés : Démarche d’investigation, secondaire qualifiant, sciences expérimentales

\section{Introduction}

Aujourd'hui, tout le monde est conscient que l'enseignement actuel des sciences ne donne pas les résultats escomptés. Il ne s'agit plus de former uniquement des scientifiques mais aussi de permettre une acculturation scientifique de citoyen vivant dans un monde où les sciences et les technologies ont une place prépondérante. Pour répondre à ces exigences, les travaux scientifiques se multiplient tant au niveau national qu'international.

En effet, les chercheurs affirment de manière quasi unanime, la nécessité d'un renouvellement de l'enseignement des sciences. Ainsi, plusieurs rapports argumentent l'exigence de repenser l'enseignement scientifique en s'appuyant sur l'investigation : d'une part pour rendre plus attractive l'image de la science et pour encourager les jeunes à s'orienter vers les études scientifiques (High Level Group, 2004 ; Rocard et al., 2007) et d'autre part pour changer les approches pédagogiques jugées trop cloisonnées (Rolland, 2006) et trop déductives (Bach, 2004 ; Rocard et al., 2007). (Coquidé et al., 2009) . 
Parmi les démarches éducatives incitantes à un apprentissage efficace, la plus en vogue actuellement est la démarche d'investigation (DI). Il s'agit d'une démarche scientifique fondée sur le questionnement et sur l'investigation et où l'apprenant est placé au centre de l'action de ses apprentissages, s'interroge, agit de manière raisonnée et communique pour construire son apprentissage tout en étant acteur des activités scientifiques (Darley, 2007). D’après André Giordan (1999), la démarche scientifique consiste à «faire émerger des éléments observables ou quantifiables, de les confronter à des hypothèses, de pouvoir maîtriser la démarche pour éventuellement la reproduire et de pouvoir discuter tous les résultats ».

L'enseignement des sciences fondé sur l'investigation n'est pas une nouveauté, que cela soit en France ou dans le monde (Lebeaume, 2011). En effet, cette méthode scientifique a été intégrée depuis plus d'un siècle, dans les curriculums des sciences dans de nombreux pays notamment les pays anglophones comme l'Angleterre et les Etats Unis (Hasni et Bousadra, 2016). En France, située dans la continuité de l'approche de la démarche scientifique et initiée avec « La Main à la Pâte » en 1996 (Coquidé et al., 2009), les démarches d'investigation ont été progressivement intégrées. En effet, la DI a été adoptée à l'école primaire en 2000, au collège en 2008, au lycée professionnel en 2009 et dans les classes de seconde générale en 2010 (Grangeat, 2014).

Les démarches d'investigation ont été déclinées, mises en texte et en œuvre de manières différenciées. Ainsi, par exemple aux États-Unis (avec diverses versions, Inquiry-Based Science Education: IBSE ; Inquiry-Based Instruction: IBI ; Inquiry-Based Teaching: IBT) et en France, ils utilisent le terme de démarche d'investigation. Ces déclinaisons variées peuvent certainement être mises en relation, au moins partiellement, avec des références historiques et culturelles distinctes (Calmettes \& Matheron, 2015).

Le nombre de travaux et de recherches consultables sur l'enseignement des sciences fondé sur l'investigation est considérable. Toutefois, dans cette étude, l'accent est mis plus particulièrement sur l'enseignant qui est la pierre angulaire de toute réforme éducative. En effet, l'enseignant joue un rôle primordial dans la démarche d'investigation car c'est lui qui doit proposer et mettre en place une pédagogie (socio)constructiviste permettant aux élèves de construire et intégrer les nouveaux savoirs (Labédie \& Amossé, 2001).

La contribution de l'enseignant dans l'application de la DI dans les classes a été traduite dans un ensemble de recherches scientifiques qui portait à la fois sur son rôle, ces conceptions épistémologiques et ses pratiques concernant cette démarche. En effet, dans l'étude de Deryter (2012), l'auteur s'est intéressé au rôle du maître dans les différentes phases de la démarche 
d'investigation et les travaux réalisés par Calmettes (2009) ont fait apparaitre des tensions et des écarts entre la façon de la représentation de la DI dans les classes de sciences physiques et la façon dont elle s'exprime à travers la demande institutionnelle. D’autre part Hirn (1995) désigne les enseignants comme des «transformateurs d'intentions didactiques ». Dans son étude l'introduction à l'Histoire de la Biologie, Giordan (1987) a établi un parallélisme entre les conceptions des enseignants et leurs conséquences pédagogiques. Dans le même contexte, une autre étude menée par Orlandi (1994) a mis en évidence le parallélisme entre les démarches pédagogiques et les conceptions des enseignants relatives à la démarche expérimentale. Enfin, l'ensemble de ces travaux insistent sur le rôle fondamental dans la mise en œuvre et l'application de la DI en classe.

Dans le contexte marocain, la rénovation des méthodes de l'enseignement des sciences est l'un des objectifs majeurs qui doivent être réaliser dans le secteur éducatif, dans cette perspective, le Maroc a ressentis la nécessité d'engager un ensemble de réformes du système éducatif (Chafiqui \& Alagui, 2013), visant la généralisation de l'enseignement, l'amélioration de sa qualité y compris celle du contenu pédagogique et la restructuration des cycles de l'éducation (Commission Spéciale d'Education et de Formation, 1999).

Cependant, la question qui se pose est: les enseignants sont-ils prédisposés à appliquer et adopter la DI dans l'enseignement des sciences expérimentales (Sciences de la vie et de la terre, Sciences de la physique et de la chimie)?

Afin de répondre à cette question, nous avons adopté la méthodologie décrite ci-dessous.

\section{Matériel et Méthodes}

L'outil de base de cette étude est une enquête sous forme d'un questionnaire suivi des entretiens semi-dirigés. L'élaboration du questionnaire s'est faite en deux étapes : dans la première, le questionnaire se composait de 10 questions dont certaines étaient des questions ouvertes demandant aux enseignants de s'exprimer sur la DI. Les données collectées étaient très minimes car les enseignants ont manifesté une résistance qu'ils justifiaient par la longueur du questionnaire. Cependant, via les entretiens, nous avons pu déduire qu'ils n’ont pas voulu s'engager dans les réponses car la terminologie Démarche d'investigation leur semble inconnu et qu'ils ne sont pas familiarisés avec ce terme. Après une discussion sur la thématique avec un groupe d'enseignants, le questionnaire a été repris et nous avons limité le nombre de questions et simplifié leur formulation.

L'étude a été réalisée dans plusieurs lycées publics de la ville de Fès (Maroc), durant l'année scolaire 2015-2016. Notre population ciblée est 
constituée de 45 professeurs de l'enseignement secondaire qualifiant. Les questions formulées se portent sur deux thèmes:

1. Représentations des enseignants sur la démarche d'investigation

2. Adoption ou non de la démarche d'investigation par les enseignants et sa mise en pratique dans la classe.

\section{Résultats et discussion}

1. Représentations des enseignants sur la démarche d'investigation

- Question1: Avez-vous déjà entendu parler de la démarche d'investigation?

Selon les réponses des enseignants à cette question (Q1), 61\% des enseignants enquêtés affirment avoir déjà entendu parler de la démarche d'investigation, à l'opposé on a 37\% des enseignants qui ont exprimé leur ignorance totale et une minorité de $2 \%$ des enseignants n'a pas donné de réponses pour cette question (Figure 1).

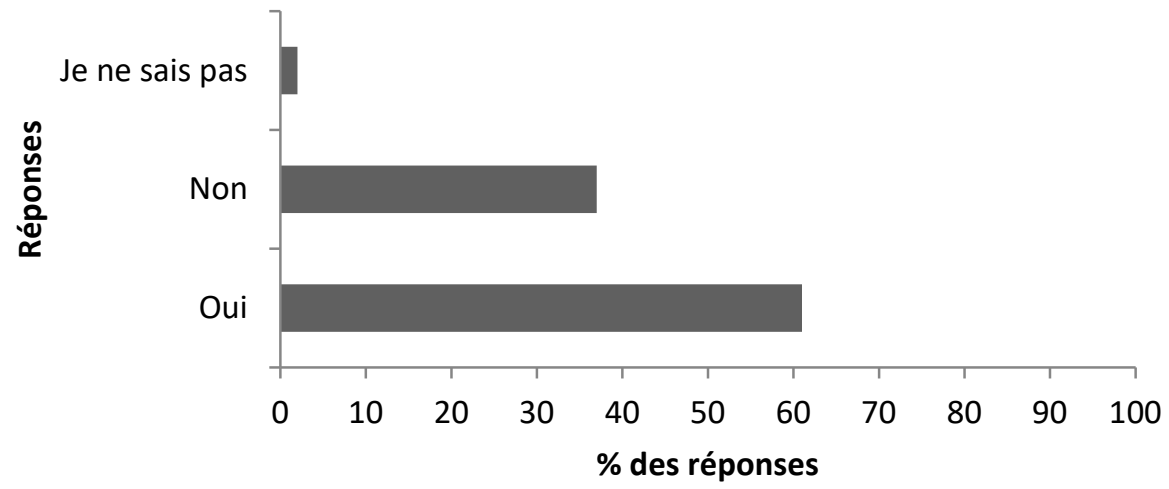

Figure 1 : Pourcentage des réponses des enseignants s’ils ont déjà entendu parler ou pas de la démarche d'investigation.

- Question 2: Donnez la définition convenable de la démarche d'investigation.

Dans cette question (Q2), on a demandé aux enseignants de choisir la bonne réponse concernant la définition de la DI, on a constaté une hétérogénéité de réponses. En en effet une majorité de 52\% d’enseignants a opté pour la réponse stipulant que la DI est une démarche basée sur l'investigation concernant les obstacles d'apprentissages des élèves et $20 \%$ des enseignants ne connaissent pas la réponse pour cette question. En revanche, seul un pourcentage de $28 \%$ a choisi la réponse la plus correcte à savoir que la démarche d'investigation est une démarche scientifique fondée sur le questionnement et sur l'investigation, mettant l'apprenant au centre de ses apprentissages lors de l’enseignement des sciences (Figure 2). 


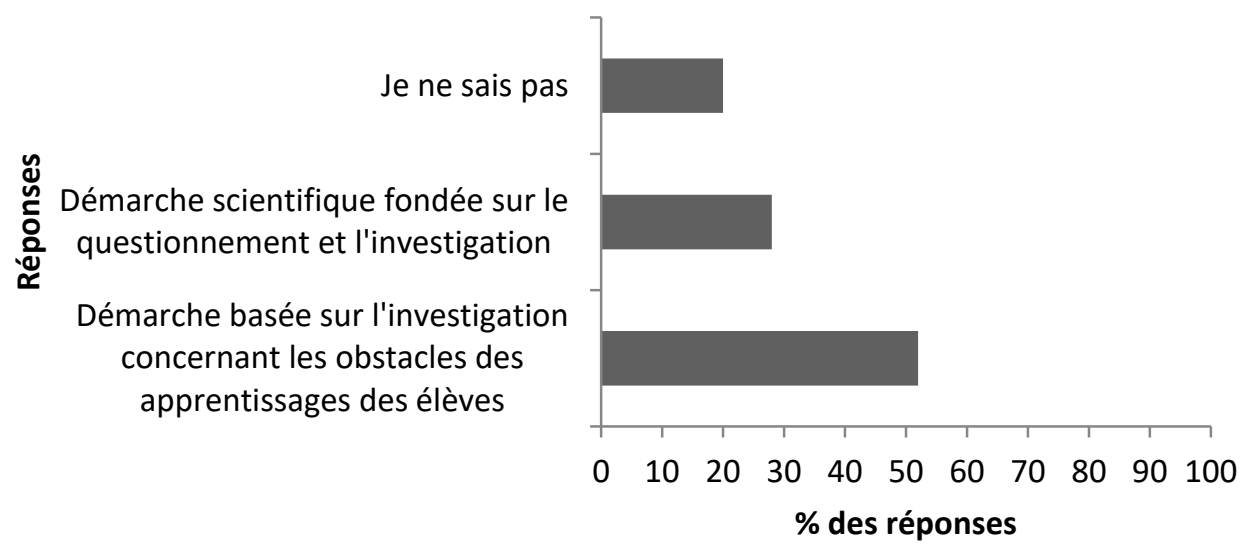

Figure 2: Pourcentages des réponses des enseignants selon l'exactitude de leur définition de la démarche d'investigation.

- Question 3: Avez-vous déjà bénéficié d'une formation continue concernant la démarche d'investigation?

Pour cette question (Q3), on a constaté que $80 \%$ des enseignants n'ont pas eu de formation sur la démarche d'investigation avant sa mise en place en pratique. La proportion des enseignants qui ont affirmé avoir déjà bénéficié d’une formation en ce sens ne dépassent pas 20\% (Figure 3).

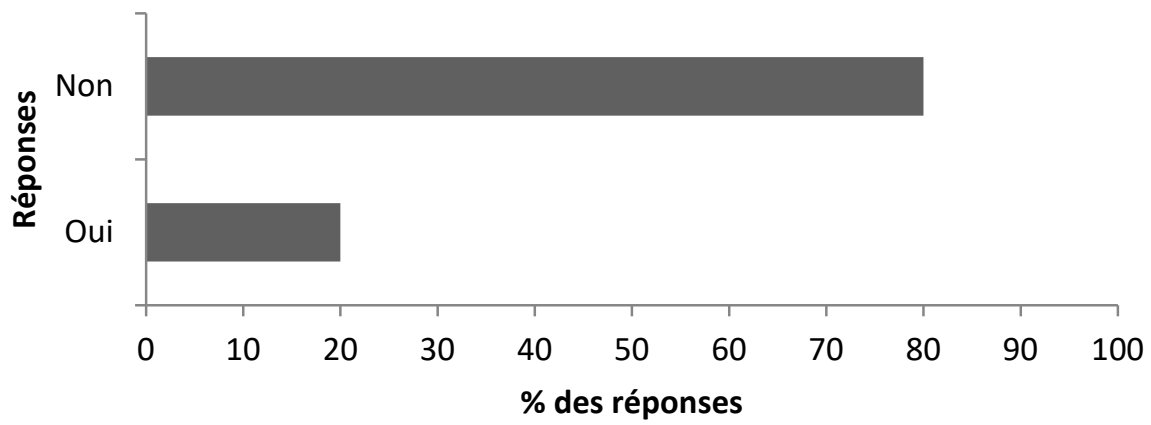

Figure 3 : Pourcentages de la répartition des enseignants selon qu’ils ont eu une formation continue sur la démarche d'investigation ou non.

En analysant les résultats issus des réponses des enseignants sur les questions Q1, Q2 et Q3 concernant les représentations de ces derniers sur la démarche d'investigation, il nous semble bien que la majorité de ces enseignants ont des confusions relatives à la démarche d'investigation puisque la majorité d'entre eux (52\%) a opté pour la formulation incorrecte 
stipulant que la DI est une démarche basée sur l’investigation concernant les obstacles d'apprentissages des élèves et cela malgré que la majorité d'entre eux (61\%) affirme avoir déjà entendu parler de cette démarche.

La présence des ces confusions chez ce groupe d'enseignants peut être liée à l'absence de la formation continue sur cette démarche ce qui s’influence sur la façon dont ils identifient la démarche d'investigation. Ces résultats se corrèlent avec d'autres obtenus par l'étude de Coupaud (2014) qui montre que les enseignants de différentes disciplines (Sciences de la vie et de la terre, Sciences physiques et chimiques, Technologie) perçoivent parfois différemment la notion de la démarche d'investigation.

Ces confusions préalables à la démarche d'investigation chez cette population d'enseignants révèlent que le système de formation à l'enseignement des sciences au Maroc ne semble pas évoluer en dépit des intentions de réforme manifestées concernant l'amélioration de l'enseignement des sciences (Madrane et al., 2009) , c'est pour cela une clarification du concept de la démarche d'investigation est nécessaire afin d'aider l'enseignant à bien comprendre cette démarche pour la pratiquer dans les bonnes conditions.

2. Adoption ou non de la démarche d'investigation par les enseignants et sa mise en pratique dans la classe.

- $\quad$ Question 4: Est ce que vous adoptez la démarche d'investigation dans votre enseignement?

D’après les données recueillies pour cette question (Q4), on a $62 \%$ des enseignants affirment qu'ils n'adoptent pas la DI dans leurs enseignements et cela par opposition à 38\% des enseignants interrogés qui avouent avoir recourir à cette démarche. Malheureusement, cela ne nous donne aucune indication sur la pratique réelle de cette démarche en classe (Figure 4).

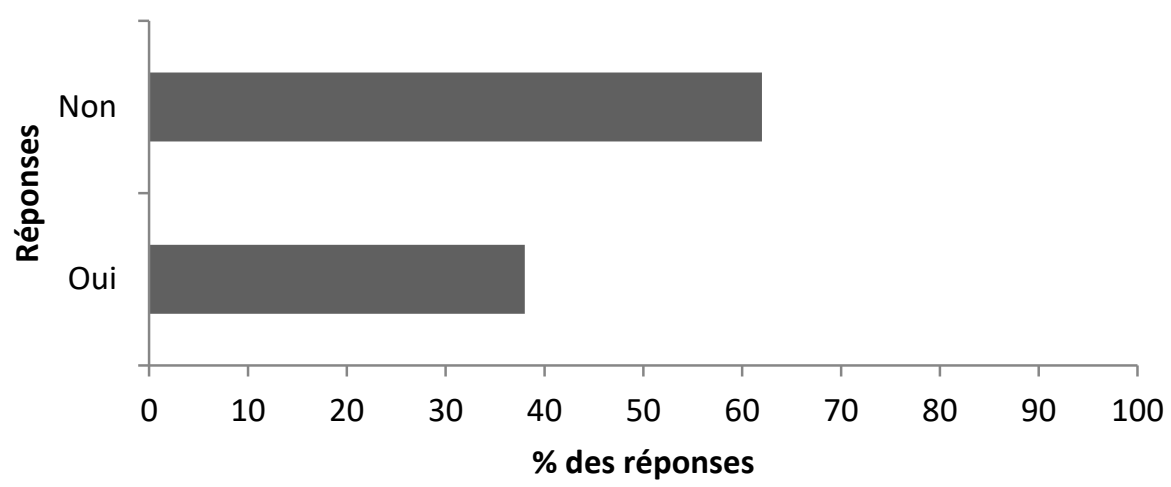

Figure 4 : Pourcentages des réponses des enseignants concernant l'adoption ou non de la démarche d'investigation. 
- $\quad$ Question 5: Quelles sont les contraintes qui empêchent l'adoption de la démarche d'investigation?

Parmi les réponses à cette question sur les contraintes limitant l'adoption de la DI en classe évoqués: la surcharge du nombre des élèves, le programme chargé et le manque d'autonomie des élèves ; ces réponses sont cités par 57\% des interrogés (Figure 5).

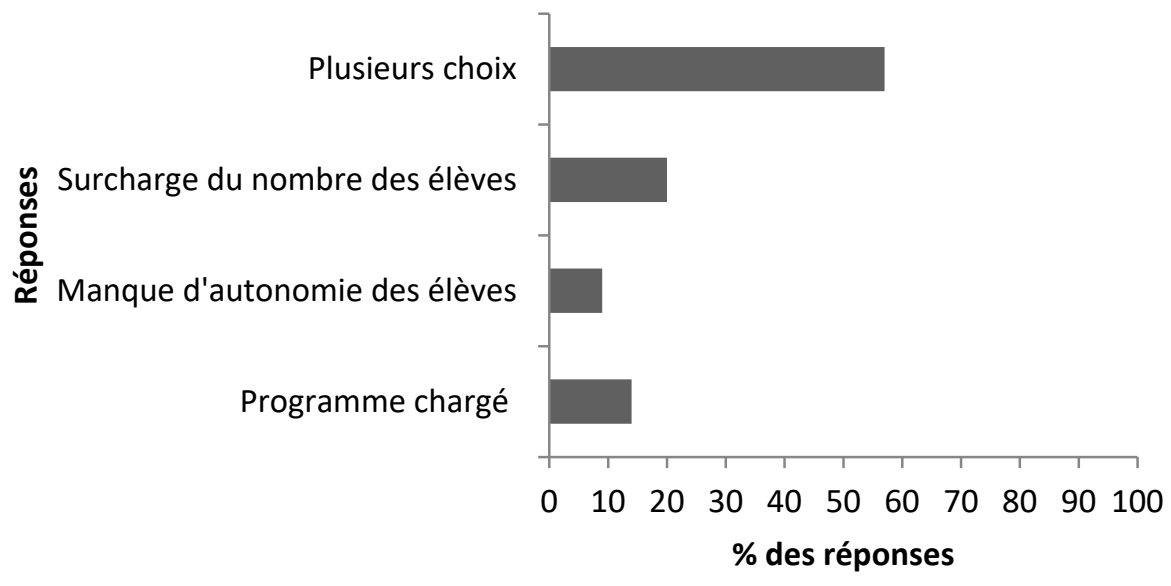

Figure 5: Pourcentages des réponses des enseignants concernant les contraintes qui empêchent l'adoption de la démarche d'investigation.

Concernant le deuxième axe de ce travail, l'analyse qualitative des résultats obtenus des questions Q4 et Q5, montre que cette démarche scientifique préconisée dans les instructions officielles ne se met pas en place sans difficultés et sans contraintes, cela est bien confirmé dans les réponses des enseignants. Chaque enseignant doit s'adapter, avec toute son individualité et ses représentations, à la mise en place de cette démarche scientifique. Les difficultés majeures concernent la surcharge du nombre des élèves, le manque d'autonomie des élèves et le programme chargé.

\section{Conclusion}

L'analyse de ce travail nous a permis de dégager un ensemble de contraintes et de difficultés qui selon les enseignants interrogés entravent l'adoption de la démarche d'investigation dans le secteur éducatif marocain. On peut citer : le programme chargé, les effectifs élevés des élèves et le manque d'autonomie des ces élèves. A cela s'ajoute l'absence de la formation continue des enseignants concernant cette démarche ce qui influence leurs représentations et la façon dont ils perçoivent cette méthode scientifique.

Ce constat met en évidence l'importance de la formation continue des enseignants. En effet, celle permet à ces derniers d'évoluer et se sensibiliser aux nouvelles approches pédagogiques. 


\section{References :}

1. Bach, J. F. (dir.). (2004). Groupe de relecture des programmes du collège. Pôle des sciences. Ministère de la Jeunesse, de l'Education nationale et de la Recherche. http://pedagogie.ac-amiens.fr/assoudppc/IMG/pdf/Rapport_Bach.pdf. In M. Coquidé, C. Fortin, \& G. Rumelhard. (2009). L'investigation : fondements et démarches, intérêts et limites .Aster No. 8 (pp. 49-76).

2. Calmettes, B. (2009). Démarche d'investigation en Physique. Des textes officiels aux pratiques de classe. Spirales (pp. 139-148).

3. Calmettes, B., \& Matheron, Y. (2015). Les démarches d'investigation : utopie, mythe ou réalité ?. Recherches en Education, No. 21.

4. Chafiqi, F., \& Alagui, A. (2013). Réforme éducative au Maroc et refonte des curricula dans les disciplines scientifiques. Carrefours de l'éducation 2011/3 (HS n 1) (pp. 29-50).

5. Commission Spéciale d'Education et de Formation. (1999). Charte Nationale d'Education et de Formation. http://www.men.gov.ma/sites/fr/Lists/Pages/charte.aspx.1999.

6. Coquidé, M., Fortin, C., \& Rumelhard, G. (2009). L'investigation : fondements et démarches, intérêts et limites. Aster No. 8 (pp. 49-76).

7. Coupaud, M. (2014). Démarches d'investigation dans l'enseignement de sciences et de technologie: perception et appropriation par les enseignants de collège. Mémoire de master en sciences de l'éducation non publié, Aix Marseille Université, Marseille.

8. Darley, B. (2007). La démarche d'investigation et son vocabulaire. IUFM d'aquitaine et DAESL Université Bordeaux2. Publié dans Grand N no 79 (pp. 99-111).

9. Deryter, S. (2012). Le rôle de l'enseignant dans la démarche d'investigation. Education <dumas-00736408>.

10. Giordan, A. (1999). Une didactique pour les sciences expérimentales. Edition Belin (p. 48).

11. Giordan, A. (1987). Histoire de la Biologie. Lavoisier, 2tomes.

12. Grangeat, M. (2014). Connaître les principes des démarches d'investigation : http://webcom.upmfgrenoble.fr/sciedu/evacodice.

13. Hasni, A., \& Bousadra, F. (2016). Les démarches d'investigation scientifique dans les pratiques d'enseignements du secondaire au Québec: défis théoriques et pratiques. Présenté dans le $84^{\mathrm{e}}$ congrès de l’ACFAS, Montréal, Canada.

14. Hassouni, T., Ameziane, N., Iraqui Houssaini, W., Lamri, D., El Madhi, Y., \& BenHaiba, R. (2014). Place de la démarche 
d'investigation dans l'enseignement des sciences de la vie et de la terre aux collèges. European Scientific Journal, Vol.10, No.22.

15. High Level Group (2004). Increasing human resources for science and technology in Europe.EC conference Europe needs more scientists. Commission Européenne. Direction de la recherche. http:/ec.europa.eu/research/conferences/2004/sciprof/index_en.html. In M. Coquidé, C. Fortin, \& G. Rumelhard. (2009). L’investigation : fondements et démarches, intérêts et limites .Aster No. 8 (pp. 49-76).

16. Hirn, C. (1995). Comment les enseignants de sciences physiques lisent-ils les intentions didactiques des nouveaux programmes d'optique de classe de quatrième ?. Didaskalia (pp. 39-54).

17. Labédie, G., \& Amossé, G. (2001). Constructivisme ou socioconstructivisme. Recuperado de http:/www.phludwigsburg.de/html/2b-frnz-s01/overmann/glossaire/constructivismeetsocioconstructivisme.doc.

18. Lebeaume, J. (2011). L'investigation pour l'enseignement des sciences : actualité des enjeux. In M. Grangeat, Les démarches d'investigation dans l'enseignement scientifique Pratiques de classe, travail collectif enseignant, acquisitions des élèves (p. 19-34). Lyon: Ecole Normale Supérieure.

19. Madrane, A., Khaldi, M., \& Talbi, M. (2009). Un système de formation à l'enseignement des sciences dans le contexte éducatif marocain: Des Conditions Pour Une Evolution Possible.

20. Orlandi, E. (1994). Les conceptions d'enseignants de biologie à propos de la démarche expérimentale. In Giordan, A., Girault, Y., \& Clément, P., Conceptions et connaissances (pp. 133-143). Berne : Peter Lang.

21. Rocard, M., Csermely, P., Jorde, D., Lenzen, D., WalbergHenriksson, H., \& Hemmo, V. (2007). L'enseignement scientifique aujourd'hui : une pédagogie renouvelée pour l'avenir de l'Europe. Commission Européenne, Direction de la Recherche. http://www.inrp.fr/vst/Rapports/DetailEtude.php?\&id=674. In M. Coquidé, C. Fortin, \& G. Rumelhard. (2009). L'investigation : fondements et démarches, intérêts et limites. Aster No. 8 (pp. 49-76).

22. Rolland, J. M. (2006). L'enseignement des disciplines scientifiques dans le primaire et le secondaire. Commission des affaires culturelles, familiales et sociales. Assemblée nationale. http://www.assemblee-nationale.fr/12/rap-info/i3061.asp. In M. Coquidé, C. Fortin, \& G. Rumelhard. (2009). L'investigation : fondements et démarches, intérêts et limites. Aster No. 8 (pp. 49-76). 